\title{
Philosophiques
}

\section{Réponse à Éric Grillo}

\section{Martin Montminy}

Volume 27, numéro 1, printemps 2000

Le matérialisme contemporain

URI : https://id.erudit.org/iderudit/004958ar

DOI : https://doi.org/10.7202/004958ar

Aller au sommaire du numéro

Éditeur(s)

Société de philosophie du Québec

ISSN

0316-2923 (imprimé)

1492-1391 (numérique)

Découvrir la revue

Citer cet article

Montminy, M. (2000). Réponse à Éric Grillo. Philosophiques, 27(1), 203-206.

https://doi.org/10.7202/004958ar d'utilisation que vous pouvez consulter en ligne.

https://apropos.erudit.org/fr/usagers/politique-dutilisation/ 


\title{
Réponse à Éric Grillo
}

\author{
MARTIN MONTMINY \\ montminy@uottawa.ca \\ Département de philosophie \\ Université d'Ottawa
}

Dans mon livre L es fondements empiriques de la signification, j'explore la question de savoir quelles sont les données empiriques pertinentes pour construire une théorie de la signification et jusqu'à quel point ces données permettent de déterminer la signification. Éric Grillo résume très bien les grandes étapes de ma démarche. Il émet toutefois des réserves concernant certaines des thèses que je défends. J e vais brièvement présenter ces thèses et montrer qu'elles ne sont pas affectées par les critiques de Grillo.

\section{Empirisme strict et empirisme modéré}

G rillo affirme déceler deux versions de l'empirisme dans mon livre, qu'il appelle respectivement « empirisme strict » $(E M)$ et « empirisme modéré » (ES). Ces deux thèses affirment :

(EM ) Une théorie sémantique légitime devrait lier tout énoncé à propos de la signification à des données empiriques en principe accessibles.

(ES) Les questions fondamentales en épistémologie et en métaphysique ne peuvent s'appuyer sur une notion bien définie de signification.

Grillo tient EM pour peu contestable, mais estime queES est problématique. La difficulté n'est pas que EM et ES sont incompatibles. (Les expressions qu'il choisit pour désigner les deux thèses sont donc quelque peu trompeuses, mais passons.) En fait, Grillo soutient que EM n'entraîne ES que si I'on admet un point de vue extrêmement limité sur la nature des données empiriques acceptables pour fonder la théorie sémantique. J'aborderai cette question à la prochaine section. J'aimerais pour l'instant expliquer pourquoi j'affirme que ES ne remet pas en question nos capacités à communiquer et à nous comprendre mutuellement.

Considérons la situation dans laquelle un tenant de la théorie relativiste (appelons-le Albert) doit interpréter quelqu'un qui adhère à la mécanique classique (appelons-le Isaac) ${ }^{1}$. À quoi réfère le mot « masse» tel qu'utilisé

1. Cet exemple s'inspire de la discussion de Field, 1973. Voir M ontminy (1998, p. 177182) pour plus de détails et pour d'autres exemples d'indétermination de la vérité. 
par Isaac? II semble qu'il y ait (au moins) deux réponses possibles : ce mot peut référer à la masse relativiste ou à la masse au repos. Si tel est le cas, il y a indétermination de la traduction de la langue d'Isaac dans celle d'Albert ; plus précisément, il y a cequej'appelle « indétermination de la vérité », puisque selon l'interprétation choisie, l'énoncé « La masse est invariante d'un système inertiel à un autre », accepté par Isaac, sera interprété par Albert comme étant vrai ou faux. L'indétermination empêche-t-elle Albert de comprendre Isaac? Certainement pas. Si Albert connaît les principes centraux de la théorie d'Isaac, il peut connaître les dispositions verbales de ce dernier, et cela est en principe suffisant pour le comprendre : le choix particulier d'un manuel de traduction n'ajoute rien à sa compréhension d'Isaac. II n'est donc pas nécessaire de poser l'existence d'une signification qui serait communiquée d'Isaac à Albert (ou de « quelque chose qui a été rendu commun », sel on les mots de Grillo) lorsque celui-ci comprend les énoncés de celui-là. La morale est qu'une notion déterminée de signification n'est pas requise pour expliquer la communication et la compréhension mutuelle, et les affirmations alarmistes de certains commentateurs de Q uine concernant les conséquences absurdes ou désastreuses de l'indétermination de la traduction ne sont nullement justifiées.

Quelles sont donc les conséquences de l'indétermination? Les mêmes que celles qui découlent du rejet de la distinction analytique-synthétique. Ces conséquences peuvent être succinctement exprimées par ES, qui affirme qu'il est vain de tenter de justifier une position sur le statut épistémologique de certains énoncés ou sur la nature de certaines entités ou propriétés du monde en se fondant sur une notion déterminée de signification. Considérons encore une fois l'énoncé « La masse est invariante d'un système inertiel à un autre » accepté par Isaac. S'agit-il d'un énoncé analytique, c'est-à-dire vrai uniquement en vertu de la signification? Si les remarques qui précèdent sont justes, cette question n'a pas de réponse déterminée. Ainsi, tenter d'établir le statut épistémologique de cet énoncé (est-il un énoncé a priori, c'est-à-dire un énoncé pouvant être justifié simplement en s'appuyant sur la signification et indépendamment des faits?) est anal ogue à tenter de découvrir des sorcières : les soi-disant preuves ou découvertes sont sans fondement.

\section{Signification cognitive et communication}

Q uine s'appuie sur les dispositions des locuteurs à accepter ou refuser des phrases dans diverses circonstances observables (ci-après, les dispositions verdictives) pour rendre compte de la signification de ces phrases ou, pour être plus précis, de ce que l'on pourrait appeler leur «signification cognitive ». (J e vais suivre ici l'usage en vigueur dans mon livre et employer le mot « signification » pour désigner la signification cognitive.) Comme le souligne $\mathrm{G}$ rillo, les données relatives aux dispositions verdictives ne permettent d'expliquer que l'un des multiples aspects de la communication entre 
locuteurs. Si l'on désire une explication complète dela communication, il faut aussi tenir compte (entre autres choses) des données relatives à l'aspect illocutoire (ou force) des énoncés. Un compte rendu de la signification d'un énoncé donné ne nous dit pas s'il s'agit d'une promesse, d'une question, d'un ordre, etc. II va donc de soi que la signification ne peut suffire à expliquer tous les aspects de la communication, et jen'affirme certainement pas le contraire dans mon livre. Tout ce que je propose, c'est de me concentrer sur la question plus restreinte de la composante cognitive de la signification.

Je ne vois toutefois pas en quoi ces considérations remettent en question $E S$ et la thèse de l'indétermination de la traduction. G rillo suggère qu'une théorie de la signification peut être enrichie de données relatives à la composante illocutoire, et que celles-ci permettent en fait de lever (en partie) les indéterminations admises par les interprétations construites sur la base des seules dispositions verdictives. J e n'aborde pas vraiment cette question dans mon livre, mais il me semble douteux que cela soit le cas. Voyons pourquoi.

Tout d'abord, il n'est certes pas évident qu'une théorie de la force pourrait être formulée indépendamment d'une théorie de la signification. M ettons toutefois ce problème de côté, et supposons qu'il soit possible de rendre compte de la composante illocutoire sans présupposer une connaissance de la signification. Supposons donc que les diverses attitudes associées à la promesse, l'ordre, l'interrogation, etc. puissent être identifiées indépendamment de la signification des énoncés impliqués, autrement dit, que ces attitudes soient en quelque sorte du même type que l'attitude (davidsonienne) de tenir un énoncé pour vrai. II serait par exemple possible de déterminer qu'en produisant l'énoncé $S$, le locuteur pose une question, sans avoir à connaître la signification de $\mathrm{S}$. La question est de savoir si l'identification des différents actes de langage accomplis par les locuteurs autorise des distinctions de signification plus fines que celles que permettent d'établir leurs dispositions verdictives. Je n'ai rien vu dans le commentaire de Grillo qui motive une réponse affirmative à cette question.

$M$ es réserves à l'endroit de la suggestion de Grillo pourraient être exprimées de la façon suivante : en quoi le fait de savoir que le locuteur nous pose une question lorsqu'il produit $\mathrm{S}$ nous éclaire-t-il sur la signification de S? Grillo pourrait peut-être proposer d'exploiter ce genre de données de la même façon que Davidson exploite les données relatives à l'attitude de tenir des énoncés pour vrais. Ainsi, pourrait-il souligner, savoir que le locuteur pose une question en énonçant $\mathrm{S}$ nous permet d'inférer un certain nombre de choses sur son état d'esprit, par exemple qu'il ne sait pas si S est vrai. La difficulté est que rien n'indique que ce genre d'information ne peut aussi être obtenu sur la base des dispositions verdictives du locuteur : on peut en effet inférer que le locuteur ne sait pas si $S$ est vrai à partir de sa disposition à donner un verdict d'abstention si on lui propose $S$. En général, un locuteur qui accomplit un certain acte illocutoire possède aussi des dispositions verdictives qui peuvent nous informer sur les présuppositions de cet acte (et sur 
bien d'autres choses). G rillo semble sous-estimer la richesse des données fondées sur les dispositions verdictives.

Ces considérations peuvent être illustrées à l'aide de l'ex emple du locuteur français du Sud-O uest, dont l'usage de l'expression « J e te promets » ne correspond pas au nôtre. II importe de noter qu'on peut aisément voir dans ce cas que le manuel de traduction homophonique doit être rejeté simplement en considérant les dispositions verdictives de ce locuteur concernant les phrases impliquant cette expression. J'ajoute tout de suite qu'il ne faudrait pas penser que l'exclusion du manuel de traduction homophonique élimine toute forme d'indétermination. Comme j'ai essayé de le montrer dans mon livre, l'indétermination de la traduction est liée à l'absence de critère permettant de séparer complètement la signification de la croyance (factuelle) ; G rillo n'a pas montré en quoi le recours à la composante illocutoire permettait d'effectuer cette séparation. L'aisance avec laquelle nous pouvons exclure certaines traductions inadéquates dans des cas comme celui du locuteur du Sud-O uest est faussement rassurante.

\section{Références}

Field, H., 1973. "Theory Change and the Indeterminacy of Reference », Journal of Philosophy, 70, p. 462-481.

Montminy, M., 1998. Les fondements empiriques de la signification, M ontréal/Paris, Bellarmin/Vrin. 